\title{
LA SENDA HACIA UNA POLÍTICA TRANSVERSAL DE INTEGRACIÓN DE INMIGRANTES EN CASTILLA-LA MANCHA ${ }^{1}$
}

\section{THE PATH TOWARDS A CROSS-SECTIONAL POLICY OF INTEGRATION OF IMMIGRANTS IN CASTILLA-LA MANCHA}

Antidio Martínez de Lizarrondo Artola

Universidad Pública de Navarra. España/Spain antidio.martinez@unavarra.es, antidio2003@yahoo.es

Recibido/Received: 28/04/09

Aceptado/Accepted: 25/08/09

\section{RESUMEN}

En este artículo se valora la construcción de políticas de integración de inmigrantes en Castilla-La Mancha. La mayoría de las comunidades ha apostado por la elaboración de planes en donde plasman estas políticas, pero esta comunidad aún no dispone de un plan transversal de integración de inmigrantes. Pese a ello, esta región va estableciendo ciertas líneas de actuación a través de planes sectoriales y de otros en colaboración con el Estado. El número y el perfil de las personas extranjeras es diferente en cada provincia de esta comunidad. Las políticas deben adecuarse a cada situación y en esta línea parece que van los esfuerzos actuales.

\section{PALABRAS CLAVE}

Política social, políticas de integración, extranjeros, PECI.

\begin{abstract}
This article evaluates the building of immigrant integration policies in Castilla-La Mancha. Most of communities have chosen the development of plans where these policies are reflected, but this community still lacks a transversal plan for the integration of immigrants. Despite that, this region is setting some action lines through the sectorial plans and others in cooperation with the State. The number and profile of the foreigners is different in each province of this community. The policies must be adjusted to each situation and so it seems to be the current trend of current efforts.
\end{abstract}

\section{KEYWORDS}

Welfare policy, integration, foreigners, PECI.

\section{INTRODUCCIÓN}

Las políticas de integración para inmigrantes que se desarrollan en Castilla-La Mancha se han caracterizado hasta hace poco por una cierta ambigüedad e indefinición en sus propósitos, así como por una reestructuración paulatina de sus planteamientos debido a 
la llegada de un notable número de inmigrantes. El asentamiento en esta comunidad ha sido más tardío que en el conjunto del Estado (en el 2000 la población extranjera de Castilla-La Mancha suponía el 0,8\%), aunque a día de hoy la tasa de población extranjera se ha acercado a la media nacional y está 1,3 puntos por debajo.

En la anterior tabla se puede comprobar cómo los nacionales de los países que han protagonizado la ampliación de la UE se están instalando preferentemente, por orden, en Aragón, Castilla-La Mancha y Madrid. En el caso de esta región está protagonizado muy mayoritariamente por personas rumanas.

Tabla 1. Proporción de personas extranjeras empadronadas por comunidades en 2008

\begin{tabular}{lcccc}
\hline & Total extranjeras & UE15 & UE12 (ampliación) & Extracomunitarias \\
\hline Murcia & 15,7 & 2,4 & 1,6 & 11,8 \\
Cataluña & 14,9 & 2,2 & 1,6 & 11,1 \\
Madrid & 15,9 & 1,3 & 4,0 & 10,6 \\
Baleares & 20,8 & 8,3 & 2,2 & 10,2 \\
La Rioja & 13,7 & 1,8 & 3,7 & 8,2 \\
Comunidad Valenciana & 16,7 & 5,4 & 3,7 & 7,6 \\
Navarra & 10,4 & 1,4 & 1,9 & 7,2 \\
\hline España & $\mathbf{1 1 , 3}$ & $\mathbf{2 , 4}$ & $\mathbf{2 , 2}$ & $\mathbf{6 , 8}$ \\
\hline Canarias & 13,6 & 6,4 & 0,7 & 6,5 \\
Aragón & 11,6 & 0,8 & 5,1 & 5,8 \\
Castilla-La Mancha & $\mathbf{1 0 , 0}$ & $\mathbf{0 , 4}$ & $\mathbf{4 , 7}$ & $\mathbf{4 , 8}$ \\
País Vasco & 5,4 & 0,8 & 0,7 & 3,9 \\
Cantabria & 5,7 & 0,7 & 1,1 & 3,8 \\
Andalucía & 7,5 & 2,5 & 1,3 & 3,8 \\
Castilla y León & 6,0 & 0,8 & 2,2 & 3,0 \\
Asturias & 3,7 & 0,6 & 0,7 & 2,4 \\
Galicia & 3,4 & 1,0 & 0,3 & 2,2 \\
Extremadura & 3,2 & 0,6 & 0,8 & 1,8 \\
\hline
\end{tabular}

Fuente: Elaboración propia a partir del Instituto Nacional de Estadística (datos a 1 de enero)

Si observamos la situación por provincias se observan notables diferencias entre ellas. En términos absolutos Toledo alberga a la tercera parte de las empadronadas en la región, aunque la provincia de Guadalajara tiene la tasa de inmigración más alta. De esta forma, con el $14,4 \%$ supera incluso en tres puntos a la media estatal, que es similar a la que se da en Cuenca y Toledo. En cambio, Albacete y Ciudad Real se encuentran a más distancia. Esta desproporción en la distribución de la población extranjera no sólo es interprovincial, sino que también es intraprovincial. Dicha distribución geográfica desigual produce que haya zonas con marcada problemática derivada del fenómeno migratorio y otras, en cambio, donde la inmigración es puramente testimonial.

El perfil de la población inmigrante en esta comunidad es diverso, ya que habría que distinguir entre temporera, con mayores problemas de alojamiento provisional y de convivencia; residente, con problemas de acceso a la vivienda y a los distintos recursos sociales, y quien utiliza la movilidad geográfica para vivir en esta comunidad y trabajar en zonas limítrofes de la cuenca mediterránea o de la Comunidad de Madrid. 


\section{EL PROCESO DE ELABORACIÓN Y DISEÑO DE UNA POLÍTICA DE INTEGRACIÓN}

Castilla-La Mancha no dispone de un plan de integración de inmigrantes. Desde los albores de la eclosión inmigrante en la región se observaba la necesidad de coordinación y transversalidad en las políticas de integración. Así, Casas y Rodríguez (2001: 183-184) solicitaban la definición de una "Política Regional de Inmigración que coordine las políticas sectoriales, planifique los programas regionales e integre las diferentes actuaciones existentes". Esta necesidad se ha acrecentado con el paso del tiempo y en la actualidad hay una especie de "Plan transversal en la sombra" mediante las acciones emanadas de planes sectoriales o líneas de actuación de las consejerías en esta materia.

Estos cuatro planes son: el Plan Regional de Integración Social (PRIS) de la Consejería de Bienestar Social, el Plan Regional para la Integración Laboral de Inmigrantes (PRILI) de la Consejería de Trabajo y Empleo, el Modelo de Educación Intercultural y Cohesión Social de Castilla-La Mancha de la Consejería de Educación y Ciencia, y por último, el Plan de Acción de Castilla-La Mancha para la Acogida, la Integración y el Refuerzo Educativo de Inmigrantes (PAAIRE), que se realiza mediante un convenio con el Ministerio de Trabajo y Asuntos Sociales. La circunstancia especial de este último Plan es que con sus fondos se sufragan acciones que encajan en los tres planes o estrategias anteriores.

Desde un punto de vista ideológico, la intervención que se ha llevado a cabo se puede dividir en dos periodos. El primero abarca hasta el año 2004, cuando la Junta de Comunidades de Castilla-La Mancha incluía para el tratamiento de las políticas de integración a las personas inmigrantes como posibles excluidas sociales. El elemento clave para abordar esta realidad fue el Plan Regional de Integración Social.

El II PRIS fue el primer documento que planteó al colectivo inmigrante como destinatario específico de sus políticas de integración en Castilla-La Mancha. Este criterio se siguió al entender que podían encontrarse en situación de vulnerabilidad, sobre todo si no tenían su situación legalizada. Simultáneamente a su surgimiento se produjo un debate acerca del modelo a desarrollar con la población inmigrante. Este planteamiento desembocó en la Red Regional de Atención a Inmigrantes, que con el paso del tiempo ha devenido en una seña de identidad de su configuración orgánica de funcionamiento. El PRIS finalizó en 2005 y ha sido prorrogado desde entonces.

Estimamos que enmarcar a la inmigración en esta categoría conlleva un riesgo. El efecto perverso de esta analogía radica en que al ofrecer un tratamiento basado en la hipotética exclusión, éste puede generar algún tipo de dependencia, que tampoco es deseada por los inmigrantes. Esta política se debe quizás a la elección que se hizo de la opción etnocultural como sintetizadora del hecho migratorio. Así, una característica del PRIS era su dedicación a la población extranjera con dificultades idiomáticas y culturales (Junta de Comunidades de Castilla-La Mancha, 2002).

La aprobación del Decreto 92/2004, de 11 de mayo, supuso un cambio radical de esta línea de orientación y el comienzo del segundo periodo, que se mantiene hasta ahora. A través de esta norma la Consejería de Trabajo y Empleo asumió las principales competencias en materia de integración de inmigrantes en esta comunidad. Del mismo modo que el PRIS fue la herramienta principal de la administración en el anterior periodo, en éste ha sido el PRILI, que se desarrolló en los años 2006-2007.

La importancia de este Plan radica en que se elaboró gracias al clima de concertación y diálogo social en la región y que surgió de la situación sociopolítica que 
suponían las competencias en materia de empleo transferidas desde el INEM, incluida la intermediación laboral. El eje en el que se basa este documento es el logro de la plena integración laboral de las personas extranjeras en la sociedad. De este modo, al mismo tiempo que el Gobierno estatal pasaba la gestión de la inmigración del Ministerio del Interior al de Trabajo y Asuntos Sociales, Castilla-La Mancha decidió separar la integración de inmigrantes del encaje en torno a la exclusión social y centrarlo en el mercado de trabajo como mecanismo de integración.

En lo que concierne a los otros planes citados, en tercer lugar aparece el Modelo de Educación Intercultural y Cohesión Social, aprobado por la Consejería de Educación y Ciencia en octubre de 2006. Este documento atemporal apuesta por una escuela inclusiva, por transformar las prácticas docentes y la cultura y organización escolar.

Por último, el PAAIRE ha supuesto probablemente el punto de inflexión en esta materia en la región, al igual que otros planes de parecida índole han significado un impulso similar en otras comunidades autónomas. La articulación en 2005 del Fondo de Apoyo a la Acogida y la Integración de Inmigrantes y Refuerzo Educativo (FAAIIRE) fue la primera piedra de una acción que vincula al Estado con las políticas de integración autonómicas. Su fin es dotar de financiación específica a las comunidades y los municipios para las actuaciones que desarrollan en esta materia.

En 2005 se suscribió un Convenio de colaboración entre la Consejería de Trabajo y Empleo de Castilla-La Mancha y el MTAS para el desarrollo de actuaciones de acogida e integración de personas inmigrantes dentro del Marco de Cooperación para la gestión del FAAIIRE. En 2006 y 2007 se firmó una prórroga del convenio. Las actuaciones a desarrollar por Castilla-La Mancha en el marco de estas prórrogas se recogen cada año en el PAAIRE, con un procedimiento similar para su elaboración.

El marco de cooperación del Fondo define 12 Ejes: Acogida, Educación, Servicios Sociales, Empleo, Vivienda, Salud, Infancia y Juventud, Igualdad de Trato, Participación, Mujer, Sensibilización y Codesarrollo, donde se enmarcan las actuaciones incluidas en los Planes de Acción que desarrollan las comunidades. Estos ejes coinciden con las áreas del Plan Estratégico de Ciudadanía e Integración (PECI), aprobado por el Gobierno en 2007.

En Castilla-La Mancha el PAAIRE comenzó con algunos de estos Ejes y en 2007 integró a todos, excepto Codesarrollo. Cada uno de ellos se desarrolla en medidas concretas indicando el organismo en quien recae la responsabilidad de su ejecución.

En la tabla siguiente se exhibe la distribución y el porcentaje por Ejes de la aportación que realizaron el MTAS y la Junta de Comunidades de Castilla-La Mancha al PAAIRE en el año 2007. Quién gestiona este Plan se comprueba viendo los Ejes con mayor gasto, por este orden: Educación, Empleo y Acogida-Servicios Sociales. El montante ejecutado por el área de Educación aglutina a casi el $60 \%$ de todo el PAAIRE.

\section{EL TEJIDO SOCIAL}

El tejido social tiene un peso muy específico en la realización de los programas y acciones de integración de inmigrantes, sea en las que gestionan los municipios enmarcadas en el PAAIRE o en otros planes sectoriales.

Castilla-La Mancha es pionera porque asigna a las entidades que participan en la Red Regional de Atención a Inmigrantes y a los sindicatos que conforman los Centros de Información y Orientación Laboral de Inmigrantes (CIOLI) una doble base para realizar 
sus funciones. La especialización funcional va acompañada de una gestión de los recursos mediante protocolos.

Dado el vasto territorio, cada entidad se encarga de una zona o comarca y, al mismo tiempo, la acción se deriva a la entidad que disponga de más infraestructura o recursos en esa área. Este modelo consensuado con las entidades autóctonas implicadas ha tenido un déficit: la falta de coordinación con los servicios sociales y la dificultad para erradicar fenómenos de solapamiento en determinados espacios, al mismo tiempo que hay otros en donde nadie interviene.

Tabla 2. Distribución y porcentaje por Ejes de la aportación del MTAS y de la Comunidad Autónoma al PAAIRE de Castilla-La Mancha. Año 2007

\begin{tabular}{|c|c|c|c|c|c|c|}
\hline Ejes & MTAS & $\begin{array}{c}\% \mathrm{~s} / \\
\text { total } \\
\text { MTAS }\end{array}$ & CLM & $\begin{array}{l}\% \mathrm{~s} / \\
\text { total } \\
\text { CLM }\end{array}$ & Total & $\begin{array}{c}\% \mathrm{~s} / \\
\text { Total } \\
\text { Ejes }\end{array}$ \\
\hline Acogida & $1.368 .910,0$ & 17,2 & $33.218,0$ & 0,7 & $1.402 .128,0$ & 11,2 \\
\hline Educación & $4.120 .378,0$ & 51,8 & $3.057 .018,6$ & 67,1 & 7.177.396,6 & 57,3 \\
\hline Empleo & $1.266 .942,4$ & 15,9 & $719.495,8$ & 15,8 & $1.989 .501,6$ & 15,9 \\
\hline Vivienda & $144.292,0$ & 1,8 & 0,0 & 0,0 & $144.292,0$ & 1,2 \\
\hline Servicios Sociales & $553.722,0$ & 7,0 & $461.407,0$ & 10,1 & $1.015 .129,0$ & 8,1 \\
\hline Salud & $21.404,9$ & 0,3 & $12.061,2$ & 0,3 & $33.466,1$ & 0,3 \\
\hline Infancia y Juventud & $98.024,0$ & 1,2 & $55.234,2$ & 1,2 & $153.258,2$ & 1,2 \\
\hline Igualdad de trato & $62.911,6$ & 0,8 & $35.449,4$ & 0,8 & $98.361,0$ & 0,8 \\
\hline Mujer & $54.922,1$ & 0,7 & $30.947,3$ & 0,7 & $85.869,4$ & 0,7 \\
\hline Participación & $62.717,2$ & 0,8 & $35.339,8$ & 0,8 & $98.057,0$ & 0,8 \\
\hline Sensibilización & $202.314,6$ & 2,5 & $113.951,7$ & 2,5 & $318.266,4$ & 2,5 \\
\hline Total por Ejes & $7.956 .538,8$ & 100,0 & $4.554 .123,0$ & 100,0 & $12.515 .725,3$ & 100,0 \\
\hline
\end{tabular}

Uno de los elementos más positivos del tejido social en esta comunidad es su fortaleza y la coordinación interna que posee, donde destaca la labor de la Red de Lucha contra la Pobreza y la Exclusión Social de Castilla-La Mancha (EAPN-CLM) como entidad dinamizadora de las distintas entidades sociales.

La Consejería de Trabajo y Empleo efectúa anualmente unas Jornadas en las que se reúnen representantes de municipios, Administración autonómica, entidades sociales y sindicatos para hablar sobre los proyectos de integración y su coordinación. Este hecho es poco habitual en el resto del Estado y es una herramienta muy valiosa a la hora de plantear las estrategias comunes de acción. La calibración aproximada de los logros conseguidos y de los puntos a mejorar se realiza en estas Jornadas, que se han convertido en una de las señas de identidad de esta comunidad como parte de la necesaria coordinación y evaluación de las políticas públicas en materia social.

Por su parte, la peculiaridad del entramado asociativo inmigrante es que gran parte de sus entidades están articuladas en torno a una Coordinadora de Asociaciones de Inmigrantes. Ésta agrupa a 46 asociaciones que se dedican principalmente a actividades de índole cultural y, apoyada por personas autóctonas, ha experimentado un gran impulso, la mayoría de las veces de forma autogestionada. Aun así, las asociaciones de inmigrantes 
son un actor secundario de la iniciativa social y todavía se encuentran con muchos problemas para ejercer como grupo de presión.

\section{SEGUIMIENTO, COORDINACIÓN Y EVALUACIÓN DE LAS POLÍTICAS POR LAS ADMINISTRACIONES}

La implicación de la administración central en esta materia tiene una doble vertiente: la técnica, con aspectos como la gestión de las autorizaciones de trabajo y residencia o el rol de la Inspección de Trabajo y Seguridad Social, y la política, en donde el FAAIRE y el PECI son las herramientas más relevantes.

En el nivel técnico la Oficina de Extranjeros es un órgano que agrupa a los servicios que se ocupan de las tareas de Trabajo y Seguridad Social, así como de Interior, de forma que se simplifican los trámites. Estas Oficinas sólo están implantadas en Toledo, Guadalajara y Albacete. Cabe resaltar que en estos momentos se aprecian en esta cuestión los efectos de la crisis, sobre todo en las autorizaciones de trabajo iniciales. La lista de ocupaciones de difícil cobertura se ha reducido y eso condiciona la concesión. El descenso se ha notado en la construcción y en la hostelería y es una barrera que implica que no se soliciten autorizaciones de empleo.

Al igual que se ha señalado la existencia de dos periodos marcados en la intervención de la Administración autonómica, en relación con la colaboración y la coordinación entre las administraciones regional y central hay que señalar esta misma identificación en las fechas, aunque evidentemente por otros motivos.

Hasta el año 2004 la coordinación política entre las administraciones regional y central no fue excesivamente fluida, quizás porque estaban gestionadas por partidos políticos diferentes. Estas divergencias se corroboran con el Recurso de inconstitucionalidad que la Junta interpuso contra la Ley 8/2000.

El cambio de Gobierno impulsó una modificación en la intervención del Estado, de forma que trata de incidir en las políticas autonómicas y favorecer la integración social de los inmigrantes con cierto carácter homogeneizador en el conjunto del territorio. En términos económicos el PAAIRE significó que la región pasara de recibir en 2004 del Estado 256.897 euros a 4.185.082 $€$ en el año 2005, cantidad que se ha incrementado hasta 7.672.850 € en 2007 (Laparra y Martínez de Lizarrondo, 2008).

Actualmente, en la coordinación entre el Estado y la Junta incide el clima de diálogo y consenso social que existe en la Comunidad Autónoma desde hace tiempo. La Delegación del Gobierno participa en todos los órganos establecidos con los agentes sociales y las administraciones autonómica y local que tienden a estructurar de forma adecuada la integración de inmigrantes en Castilla-La Mancha. Un ejemplo fue la constitución en 2007 de la Mesa de Inmigración y Relaciones Laborales compuesta por la Consejería de Trabajo y Empleo, los sindicatos y los empresarios. Su objetivo, entre otros, es impulsar el recién creado Foro Regional para la integración de la población inmigrante (Decreto 338/2008, de 4 de noviembre), así como crear una comisión de expertos que analice la situación socio-laboral de las personas inmigrantes en la región.

La otra vertiente de la coordinación vertical se refiere a los municipios y diputaciones. Salvo alguna excepción, las diputaciones apenas se han involucrado en acciones encaminadas a la integración de inmigrantes. En algunos casos da la impresión de 
que como se observa que otros entes administrativos implementan programas, no se actúa en la materia, con lo que esta dinámica tiende a retroalimentarse.

Respecto a los municipios, el PAAIRE ha propiciado un punto de inflexión en su rol. De esta forma, se han implicado en la gestión y ejecución de numerosos proyectos, además de aportar el $10 \%$ de la subvención recibida por cada acción. El clausulado del Convenio firmado exige la derivación de un porcentaje de la aportación económica del Ministerio de Trabajo y Asuntos Sociales a las entidades locales con mayor presión migratoria. $\mathrm{Su}$ aparición ha sido, sin duda, el mejor apoyo y refuerzo para una política transversal en esta materia e incluso se puede hablar de que, en determinados espacios y salvando las distancias, se ha convertido por su relevancia en la práctica en el propio Plan de integración de inmigrantes de la Junta de Comunidades de Castilla-La Mancha. El seguimiento y análisis de los proyectos se ha supervisado conjuntamente entre técnicos de la Administración local y la autonómica y esto es básico para que haya un control eficiente.

La característica de los proyectos desarrollados por los municipios ha sido la no estructuración de servicios específicos para inmigrantes y se ha delegado en los entes sociales el trabajo y la atención a inmigrantes. En muchos casos se orienta desde los Servicios Sociales Básicos hacia los recursos específicos de los sindicatos que conforman los Centros de Información y Orientación Laboral de Inmigrantes o hacia los recursos existentes en el municipio de la Red Regional de Atención a Inmigrantes.

No obstante, hay excepciones entre las capitales de provincia, como es el caso de Albacete. Este municipio fue en 2003 el primero que estructuró de forma independiente un Servicio Municipal de Atención Social al Inmigrante y que se enmarcaba en el Plan de inmigración de la Concejalía de Acción Social. En la actualidad, es el único que cuenta con una estructura local que engloba a los actores presentes en esta materia con la llamada Mesa de la Inmigración, que en breve se va a conformar como Consejo de Inmigración. En Albacete se encuentra además la Casa de la Solidaridad, un referente en el ámbito de las políticas de integración a nivel local en la región, que depende de la única concejalía específica de inmigración de un municipio en Castilla-La Mancha.

Un déficit es la implantación de foros y mesas locales de participación de personas inmigrantes. Estimamos que es una acción muy interesante para el desarrollo de políticas de integración en esta región, como lo ha sido en otros lugares. En general, en otras comunidades tienen el carácter de órgano consultivo en el ayuntamiento y son un punto de encuentro de los actores implicados en cada localidad para dar voz a inmigrantes en cuestiones locales, además de ser a menudo un punto de inflexión en las políticas de integración (Martínez de Lizarrondo, 2009).

En relación a la coordinación horizontal, la falta de un plan transversal en materia de integración de inmigrantes ha supuesto que la coordinación, el seguimiento y la evaluación de las políticas de integración sólo hayan incumbido a los departamentos que las han impulsado. Cada Consejería ha llevado a cabo sus propios seguimientos, pero no se han rendido cuentas ante ningún órgano porque éste no existe de una forma concreta. Hasta ahora la Consejería de Trabajo y Empleo, la Consejería de Educación y Ciencia, la Consejería de Salud y Bienestar Social, el SESCAM, el Instituto de la Mujer y el Instituto de Juventud son los únicos organismos que se han implicado en este tema.

En la actualidad se están dando pasos positivos en esta cuestión, que se concretarán más si se lleva a cabo una estrategia transversal que implique a toda la Junta. Un avance en este sentido es el Foro Regional para la integración de la población inmigrante, que esperemos sirva de dinamizador de esta estrategia. Además de este órgano, parece 
necesario que se implante con carácter permanente un Observatorio de la Inmigración, ya que sólo funcionó de forma experimental un año.

\section{INTENSIDAD DE LA COMUNIDAD AUTÓNOMA EN LA INTERVENCIÓN}

La intensidad con la que las distintas áreas de la Junta de Comunidades de CastillaLa Mancha han intervenido tiene mucho que ver con la planificación de acciones en esta materia. Por este motivo es lógico que las áreas que más se han implicado son las tres consejerías que gestionan el PAAIRE y que aprobaron documentos en sus respectivas áreas que, de un modo u otro, inscriben a las personas inmigrantes como destinatarias de las políticas de integración.

Estas consejerías y sus planes en la materia son: Consejería de Bienestar Social (Plan Regional de Integración Social), Consejería de Trabajo y Empleo (Plan Regional para la Integración Laboral de Inmigrantes) y Consejería de Educación y Ciencia (Modelo de Educación Intercultural y Cohesión Social de Castilla-La Mancha).

\subsection{Servicios Sociales}

La estructura específica más relevante que aglutina los recursos que se destinan al colectivo inmigrante es la Red Regional de Atención a Inmigrantes, que depende de la Consejería de Salud y Bienestar Social. Su desarrollo es positivo, ya que ha cumplido el objetivo de dar una primera atención al inmigrante. Es un dispositivo único en el Estado para una comunidad sin un plan en la materia (Martínez de Lizarrondo, 2008). Sin embargo, la región avanza hacia otras fases del fenómeno migratorio y parece necesario revisar el modelo y pasar a otros dispositivos más normalizados.

Una cuestión importante de la Red es su articulación en dos niveles diferenciados e interrelacionados (Servicio de Integración Social, 2008).

El primer nivel corresponde a los proyectos de intervención social cuyo objetivo es integrar a inmigrantes de una forma exclusiva, que se desarrollan en colaboración con las entidades sociales en un ámbito local o comarcal en función de las necesidades detectadas por los servicios sociales. Dicho nivel distingue estos proyectos de aquéllos dirigidos al conjunto de personas afectadas por la exclusión social, pero en los que sus factores de riesgo de exclusión no son inherentes a su condición de inmigrantes. En este nivel es donde se enmarcan más adecuadamente los proyectos integrados en el PRIS.

El segundo nivel se configura a través de diversos dispositivos de atención que por sus características y contenidos trascienden las estructuras locales y agrupan a más territorios. Lo que diferencia a este nivel son los aspectos de territorialidad, contenidos, función y homogeneización de la atención, puesto que los proyectos locales del primer nivel deben ser más heterogéneos en su configuración.

Los recursos que, con cobertura regional y organizada en red, operan en todo el territorio son los siguientes (Servicio de Integración Social, 2008): Servicio de Atención y Mediación intercultural (SAMI), Alojamiento especializado -que incluye tres tipos: las Casas de Acogida Temporal para Inmigrantes (CATI); los Pisos de atención humanitaria y acogida en la península de inmigrantes procedentes de Ceuta, Melilla y Canarias; y la Información y alojamiento para inmigrantes por razones humanitarias-, Acciones de Refuerzo ante situaciones de emergencia social, Intermediación lingüística, Intermediación 
para el acceso a la vivienda y Prevención del conflicto social. En todos ellos ha sido relevante la financiación con cargo al PAAIRE.

El principal recurso de la Red son los SAMI, que están en 70 puntos de Castilla-La Mancha y son gestionados por municipios. Hasta 2004 los SIAI, gestionados por UGT y $\mathrm{CCOO}$, desarrollaban sobre todo funciones encaminadas a facilitar la inserción laboral y ofrecer asesoramiento jurídico. Desde ese año los sindicatos realizan esas funciones en los CIOLI, mientras que los SAMI se centran en la primera acogida y se le da relevancia a la figura del mediador social intercultural. Sin embargo, este dispositivo no proporciona atención normalizada, ya que discrimina positivamente a las personas inmigrantes. En los municipios más grandes ha devenido en una vía de entrada paralela a los Servicios Sociales Básicos, ya que la persona inmigrante accede directamente al SAMI en lugar de producirse la derivación mediante protocolo a través del SIVO.

Una cuestión de difícil tratamiento es la que se circunscribe a la asistencia que se da en tres asentamientos existentes en Albacete. Este tema es un asunto de primera magnitud, tanto para las personas afectadas, en primer lugar, como a nivel mediático y vecinal. Lo ideal sería intervenir a través de una red normalizada, como se ha intentado en otras comunidades autónomas, pero para ello es evidente que es necesaria también una mayor coordinación entre áreas. La reorientación de toda la red de alojamiento, también la de emergencia, hacia pautas más normalizadas también debiera ser un eje básico de discusión porque la discriminación positiva no conduce a la integración.

Otra cuestión son recursos de adaptación para una necesidad concreta, que son positivos. La Intermediación lingüística es muy buena práctica, pero debiera ser extendida a otras Consejerías además de Salud y Bienestar Social, que son las áreas donde se desarrolla. Se trata de un dispositivo de traducción simultánea a una amplia gama de idiomas para facilitar la comunicación entre la población inmigrante y los técnicos de la Red Básica de servicios Sociales, cuando se encuentren con dificultades idiomáticas y culturales para acceder a diferentes recursos sociales. En 2007 se realizaron un total de 2.085 traducciones, que supusieron 32.268 minutos de conversación (Dirección de Trabajo e Inmigración, 2008).

La coordinación entre áreas se necesita para el desarrollo de determinados proyectos con colectivos más vulnerables, como mujeres o menores (con atención especial a zonas donde se concentra más juventud inmigrante, como el Corredor del Henares y en La Sagra). La estructuración interna de la administración y la inclusión de los proyectos en cada área requiere una coordinación transversal en estos temas entre la Consejería de Salud y Bienestar Social y otros organismos de la administración.

También parece necesaria la adecuación de los proyectos PRIS. Según EAPN-CLM (2006: 128) estos proyectos no se repartían bien en función de las necesidades básicas de los inmigrantes, no tenían un nexo con la distribución provincial de éstos y además el número de acciones era insuficiente porque "la ratio inmigrantes/acción es de $480 \mathrm{y}$, si se consideran sólo las acciones definidas como las más acuciantes para la población inmigrante, la ratio sobrepasa a los 5.760 inmigrantes por acción".

La reconducción de este modelo de acogida y, subrayamos, de cohesión social, además de mejorar la funcionalidad debe implicar una evaluación conjunta y una coordinación de todos los dispositivos. Es preciso recordar que ligado a ello aparece la especificidad territorial. El énfasis se pone en los proyectos de intervención basados en la realidad del territorio. Este hecho es positivo, pero deriva en ocasiones en la puesta en marcha de recursos para la inclusión de los inmigrantes que conllevan al mismo tiempo una falta de coordinación con el sistema público de Servicios Sociales u otras áreas. 


\subsection{Educación}

El desarrollo de las políticas de integración en esta área ha experimentado una notable evolución y en este contexto hay dos cuestiones que se complementan para dar lugar al resultado actual: la propia normativa e impulso de determinadas medidas por la Consejería de Educación y Ciencia y el apoyo económico que desde 2005 ha reportado el PAAIRE, que ha servido para ejecutar varias de estas medidas.

El Consejo Escolar de Castilla-La Mancha (2004: 37), al constatar el aumento de inmigrantes escolarizados, pedía la adopción de medidas urgentes, ya que "ante la perspectiva del aumento inminente de alumnado inmigrante, nuestro sistema educativo (igual que el del resto de Europa) no está preparado para asumir esta situación”. La serie de medidas implementadas nos hace pensar que el sistema ha respondido con relativa fluidez a este reto y que son injustificados estos temores.

A través de las medidas puestas en marcha podemos comprobar que la sociedad castellano-manchega se va comprometiendo con la integración de inmigrantes en el ámbito educativo. En este sentido, es relevante que la Consejería de Educación y Ciencia entiende que para lograr la integración plena carecen de sentido las acciones dirigidas sólo para inmigrantes, ya que se rompería el sentido de inmersión de los mismos en la sociedad de acogida y se constituirían guetos diferenciados. Pese a que el FAAIRE es en principio una inversión específica para acciones destinadas a inmigrantes, el énfasis que Castilla-La Mancha realiza en la educación como un ámbito normalizado ha incidido en que en sus acciones "no sólo han participado personas de origen inmigrante, sino que las instituciones implicadas también han desarrollado actividades dirigidas a colectivos interculturales, con el fin de favorecer la interrelación y la acogida" (Dirección de Trabajo e Inmigración, 2008: 8).

Acerca de la normativa o acuerdos que conllevan un refuerzo en la intervención con inmigrantes hemos de subrayar tres documentos aprobados entre 2006 y 2008: el Modelo de Educación Intercultural y Cohesión Social, el Acuerdo por la Convivencia en los Centros Escolares de Castilla-La Mancha y el Decreto 3/2008 de la Convivencia Escolar, que establece el marco por el que los centros han de regular la convivencia escolar y definir un conjunto de actuaciones y medidas para su promoción y mejora.

Hasta ahora la seña de la educación para inmigrantes en Castilla-La Mancha es el desarrollo de la Compensación Educativa, especialmente con las incorporaciones tardías, que son integradas directamente en el aula. Sin embargo, se han implantado otras interesantes medidas de atención a la diversidad (Dirección de Trabajo e Inmigración, 2008): los Equipos de Apoyo Lingüístico para la inmersión lingüística del alumnado que desconoce el castellano, los Planes de Actividades Extracurriculares en el Marco de las Ciudades Educadoras, el Programa de gratuidad de materiales curriculares, Becas para la Prestación del Servicio de Comedores Escolares, Proyectos Singulares en centros educativos y Programas de Innovación Educativa de educación intercultural, Formación del profesorado, Elaboración de materiales didácticos en diferentes idiomas, así como la impartición del programa de Enseñanza de la Lengua y Cultura de Origen (ELCO) a alumnos/as de Infantil, Primaria y Secundaria de origen marroquí y rumano.

\subsection{Salud}

El principio de normalización ha influido en que apenas se hayan articulado iniciativas que adecuen los recursos a la población extranjera. La cuestión básica en el área sanitaria es la tenencia por parte de la población inmigrante de tarjeta sanitaria. La evolución de este aspecto en los últimos años muestra aspectos positivos, aunque con 
matices. En 2004 el 22,8\% de los extranjeros no poseía la tarjeta sanitaria, mientras que en 2007 este porcentaje había descendido al 17\% (Comisiones Obreras de Castilla-La Mancha, 2004 y 2007). Ello no obvia que sigue siendo un porcentaje muy relevante y que habrá que arbitrar las medidas oportunas para la extensión real de la tarjeta.

A partir de 2007 se han impulsado algunas acciones que son necesarias en este ámbito. Un ejemplo es la incorporación de mediadores sociosanitarios en los centros dependientes del SESCAM para garantizar su accesibilidad a los servicios sanitarios y mejorar su relación con los profesionales que trabajan en la sanidad pública (mediante un acuerdo con Médicos del Mundo y la Universidad de Castilla-La Mancha).

Igualmente es reseñable la información que ofrece la Guía de la Dirección General de Salud Pública y Participación (2006) o los estudios que desde el ámbito universitario y el sanitario se realizan específicamente sobre población inmigrante. En este sentido, el SESCAM constituyó un grupo de investigación, en colaboración con la Universidad de Castilla-La Mancha, con el fin de realizar un diagnóstico de la salud y la enfermedad de los inmigrantes residentes en la región. Asimismo debemos subrayar un estudio del Grupo de Investigación sobre Salud Intercultural, que destierra prejuicios que advierten del colapso de la sanidad pública por inmigrantes porque demuestra que no colapsan las Urgencias, ni las consultas ginecológicas ni las salas de partos del sistema público de salud (La Verdad, 31 de julio de 2007).

\subsection{Vivienda}

Este área posee una ambivalencia, ya que hay elementos estructurales que favorecen y otros que dificultan la integración de inmigrantes. Por ejemplo, dado el menor precio de la vivienda en comparación con la media nacional, esto ha provocado un aumento del número de propietarios que son inmigrantes. Según la Encuesta Nacional a Inmigrantes del INE, casi un tercio de la población extranjera residente en la región poseía una vivienda en propiedad en 2007.

Asimismo, hay indicadores que insinúan una mejora en esta área en la calidad subjetiva del estado de las viviendas, del equipamiento y de la disminución del hacinamiento extremo, pero motivada porque se paga mucho más por el alquiler (Comisiones Obreras de Castilla-La Mancha, 2007: 273).

Ahora bien, ésta es sin duda el área más deficitaria en cuanto a recursos y programas ejecutados con vistas a la integración y cohesión social. La Consejería de Ordenación del Territorio y Vivienda no ha intervenido en esta materia e incluso las medidas de tipo normalizado que desarrolla son escasas en ámbitos fundamentales, como por ejemplo, el fomento del alquiler. En principio, el hacinamiento y las malas condiciones higiénico-sanitarias son los mayores problemas de la vivienda para las personas inmigrantes en la región. Para la Red de Lucha contra la Pobreza y la Exclusión Social en Castilla-La Mancha (2006: 117) son dos las problemáticas fundamentales: a) los asentamientos se originan porque hay personas sin ningún tipo de recursos económicos a quienes les resulta imposible alquilar una vivienda; b) el recelo y la negativa de gran parte de las personas propietarias a alquilar sus pisos a inmigrantes.

En este área se ha mantenido desde 1993 hasta 2004 una norma discriminatoria en relación a la ocupación de viviendas por inmigrantes. El artículo 14, apartado C, del Decreto 93/1993 sobre adjudicación de viviendas de promoción pública estipulaba que sólo podían acceder a viviendas de protección pública si existía un convenio recíproco con los países de origen. Dado que España no tiene suscrito ningún convenio de estas 
características con ningún país del mundo, en la práctica la población extranjera no tenía posibilidades de que se les otorgara este tipo de viviendas, ya que si una persona autóctona lo denunciaba, automáticamente la promoción de vivienda pública afectada quedaba invalidada y los ocasionales adjudicatarios extranjeros eran excluidos.

Con posterioridad, el Decreto 3/2004, de Régimen Jurídico de las viviendas con protección pública derogó el Decreto 93/1993 y siempre que cumplan los requisitos estipulados para toda la población -renta, empadronamiento, etcétera-, las personas extranjeras pueden obtener en igualdad de condiciones una vivienda de esta tipología.

En la actualidad parece necesario revisar y reestructurar, junto con los otros programas de vivienda de la Red Regional de Atención a Inmigrantes, el Programa de Intermediación para el acceso a la vivienda. Esta medida afecta únicamente a inmigrantes y no se pueden obviar las consecuencias de una medida de discriminación positiva de esta índole, ya que puede lograr el objetivo contrario al que se busca. Sin embargo, insistimos en que no debiera ser la Consejería de Salud y Bienestar Social quien debe intervenir para procurar el acceso, sino la Consejería de Ordenación del Territorio y Vivienda a través de iniciativas y proyectos que sean para toda la población.

La temporalidad es uno de los rasgos socio-laborales de Castilla-La Mancha más relevantes. La recolección en el sector agrícola propicia la llegada de inmigrantes y la contratación de personas sin permisos de trabajo en vigor, por lo que se incrementa el trabajo desregulado en esta población. Las concentraciones de temporeros generan un déficit en las estructuras de algunos pueblos y se percibe una ausencia de alojamientos habilitados y, salvo algunas iniciativas aisladas, la escasa implicación del colectivo empresarial. De esta forma, se han detectado graves problemas de infravivienda y de chabolismo en determinados pueblos con motivo de la recolección agrícola. Por ello parece fundamental la implicación de los contratadores en el mercado agrícola para proporcionar una red más amplia de la existente de alojamientos temporales para que donde trabajen las personas extranjeras tengan un sitio para dormir.

\subsection{Empleo y Formación}

La asunción de la gestión de las competencias en materia de inmigración por la Consejería de Trabajo y Empleo, la aparición del PAAIRE y la aprobación del PRILI influyeron en que este área haya tenido un notable impulso en los proyectos puestos en marcha. Una medida emblemática de este área fue la implantación de los CIOLI.

Algunas cuestiones positivas de la intervención en este área son el aumento de los autónomos y la constitución de empresas de economía social. Aún así, es necesario precisar que parte del aumento de trabajadores por cuenta propia se debe a determinadas estrategias, que en el caso de rumanos y búlgaros se debe a la moratoria de trabajo que pesó sobre ellos de 2007 a 2009. Desde este año son ciudadanos de la UE a todos los efectos y ello redunda positivamente en otras áreas que conciernen a su integración.

En nuestra opinión, las acciones planteadas afectan a la mayoría de los aspectos inherentes en un proceso de inserción laboral y conforman un camino que dará buenos frutos si realmente se aplican las perspectivas de trabajo, investigación y formación ya iniciadas. Desde hace años son corrientes el mismo tipo de peticiones de intervención en este área. Otra cuestión es que luego se lleven con eficiencia realmente a la práctica.

Comisiones Obreras (2007: 151) destaca ciertos rasgos del mercado laboral en la región: a) la mayor influencia que está adquiriendo la red social en el acceso al empleo; b) 
el descenso de la importancia de los Servicios Sociales y las ONG a la hora de insertarse; c) la mayor tasa de "sin papeles" que no puede acceder a un empleo irregular.

Desde 2004 el diálogo social ha sido un punto de partida que ha propiciado numerosas actuaciones conjuntas con el aval de las distintas administraciones. Es cierto que, si se puede hablar en términos de "integración normalizada", en las localidades con cultivos estacionales ésta es muy difícil porque hay periodos que están los inmigrantes y otros que no y eso altera los ritmos de la integración. Aun así, los problemas de convivencia que hubo en su momento no se han vuelto a dar, al menos de esa forma.

Ahora bien, la actual situación económica ya se está notando que afecta más seriamente a muchos trabajadores extranjeros y es necesario arbitrar medidas que eviten situaciones de vulnerabilidad. Muchos extranjeros, si no mantienen el mínimo de cotizaciones sociales necesarias para las renovaciones, perderán en los próximos meses los permisos de trabajo en Castilla-La Mancha. Esta cuestión puede romper la cadena que a los cinco años hace que ese permiso temporal se convierta en permanente. Estos permisos afectan personalmente a un individuo, pero indirectamente a toda una familia, por ejemplo, que pudiera devenir en situación de irregularidad. La situación es grave y algunos inmigrantes incluso solicitan información para retornar a su país, aunque luego quienes deciden tomar esa decisión siguen siendo, por diversos factores, muy pocos.

\subsection{Sensibilización}

Este ámbito es quizás donde más hincapié se debe hacer, aunque algunos parámetros indican una buena receptividad de la sociedad castellano-manchega en los últimos años. Es difícil comprobar si hay una relación causa-efecto entre las acciones puestas en marcha y la mejora en la convivencia o el sentimiento de discriminación, pero lo cierto es que hay una tendencia descendente respecto a las personas inmigrantes que se han sentido discriminadas en la región. Así, Comisiones Obreras de Castilla-La Mancha (2007: 194-196) indicaba que, a pesar de todo, el rechazo xenófobo descendía con respecto a 2003 en el trabajo, el área de vivienda o en los lugares de ocio, aunque aumentaba ligeramente en el trato con las administraciones públicas.

En los pueblos y ciudades es donde se cultivan las condiciones que facilitan o dificultan la integración social. Por ello la ejecución de todo tipo de acciones en el ámbito local que interrelacionen de manera normalizada a los residentes en ese espacio es la mejor medicina contra la intolerancia y la discriminación, ya que genera un impulso en las políticas de los municipios en materia de cohesión social.

Entre estas cuestiones, es preciso fomentar el empadronamiento de las personas extranjeras que residen en la región, ya que es el primer paso para una acción real que habilite todos los instrumentos adecuados en su proporción necesaria. En ocasiones ha habido problemas en esta cuestión en algún municipio. Quizás sea conveniente recordar el contenido de la Resolución 16.679, de 21 de julio de 1997, del INE. El artículo 4 de esta Resolución indica que cualquier persona tiene derecho a ser empadronada aunque viva en una chabola, infravivienda o incluso en ausencia total de techo y el artículo 5 insiste en la necesidad (y derecho) de los extranjeros de estar inscritos en el padrón.

Una duda en estos momentos es si la población autóctona puede ver a las personas inmigrantes como competidoras ilegítimas por los puestos de trabajo. Ello pudiera ocasionar enfrentamientos o competencia en algunos nichos laborales, con el consiguiente aumento de los prejuicios. En este sentido, la mayoría de los actores implicados estima que no hay este tipo de problemas en la región. Aun así, habrá que estar atentos a la evolución 
del desempleo y de otros factores, ya que en otras comunidades limítrofes como Extremadura se han detectado casos de "fricción laboral" en determinados sectores laborales entre población inmigrante y población autóctona poco cualificada (Consejo Económico y Social de Extremadura, 2003: 186-189).

\section{CONCLUSIONES}

En este momento la situación económica plantea unos interrogantes a nuestra sociedad que habrá que ver si es capaz de resolver: si una época de bonanza económica ha propiciado que se produzca sin estridencias la progresiva integración de la población inmigrante, es sobre todo en una época de recesión cuando debe haber estrategias inteligentes y reactivas de integración y cohesión social.

Por este motivo estimamos que debe existir una reorientación por parte de la Junta de Comunidades de Castilla-La Mancha para poner en marcha políticas holísticas de integración. La inmigración no ha hecho sino poner en evidencia las debilidades y las fortalezas estructurales del Estado de Bienestar. Castilla-La Mancha ha defendido durante el periodo analizado la normalización, pero ésta tiene un riesgo porque necesita una inversión. Sectores como los servicios sociales, la vivienda y otros necesitan una inyección de recursos humanos y/o técnicos para ofrecer servicios a todas las personas.

No debemos olvidar en estos momentos una cuestión esencial: si la inmigración ha puesto en evidencia diversas cuestiones que ya venían de lejos, la crisis es la que va a mostrar con toda su crudeza las lagunas de nuestro modelo de bienestar en el sistema de protección social. Por ello habrá que prever estrategias que eviten la exclusión, pero planteadas para toda la ciudadanía, sin distinción de nacionalidad.

El PAAIRE ha supuesto el punto de inflexión de esta cuestión. Esto no quita para que Castilla-La Mancha tenga la oportunidad de ligar la elaboración de un Plan transversal de integración de inmigrantes a la estrategia que ha planteado el Estado con el PECI. La reciente aprobación del Foro Regional para la integración de la población inmigrante en Castilla-La Mancha plantea posibilidades reales de actuación transversal en esta materia, que deben ser aprovechadas. Es necesario beneficiarse de las sinergias creadas en torno a las acciones impulsadas por el Plan de Acción para establecer una estrategia de integración que sea duradera en el medio plazo. Hay una muy buena base en la mayoría de las áreas de intervención y es preciso observar qué se hace en otros espacios, pero eso sí, con el compromiso económico para que las acciones se ejecuten.

Castilla-La Mancha debe avanzar en la reestructuración del funcionamiento de algunos dispositivos y romper con una dinámica que atiende al colectivo inmigrante de una forma demasiado especializada. Así, parece verosímil que el efecto producido es una separación. Teóricamente, aunque no exista un Plan de integración de inmigrantes, se apuesta por un modelo intercultural que debe promover la normalización.

Este modelo debe tener, según nuestra opinión, elementos de discriminación positiva sólo en las fases iniciales de ciertos servicios y con una fecha de inicio y de finalización para los mismos. Esto no ocurre en algunos casos con algún servicio de la Red Regional de Atención a Inmigrantes, donde aparecen elementos de discriminación positiva que pueden ser permanentes y que pueden ayudar a la instauración no deseada de un modelo de gestión de la diversidad cultural basado en la segregación. 
En definitiva, para la elaboración de un Plan transversal de integración de inmigrantes es posible que sea preciso vencer las resistencias en determinados ámbitos de la administración que dan la impresión de no saber cómo abordar este fenómeno y actuar empujada por los acontecimientos. La forma en que la Junta pueda aunar voluntades e implicar a algunos sectores de su propia administración y también del ámbito local, poco proclives a involucrarse activamente en las distintas iniciativas que se promueven en este ámbito, va a ser fundamental para el devenir de las políticas de integración en Castilla-La Mancha.

\section{NOTAS}

${ }^{1}$ Este artículo se basa en el estudio "Una estrategia de integración de inmigrantes para Castilla-La Mancha", que el autor ha realizado a finales de 2008 para la Red Europea de Lucha contra la Pobreza y la Exclusión Social de Castilla-La Mancha (EAPN-CLM) y la Dirección de Trabajo e Inmigración de la Consejería de Trabajo y Empleo de Castilla-La Mancha.

\section{BIBLIOGRAFÍA}

CASAS, F. Y RODRÍGUEZ, C. (2001): La inmigración económica en Castilla-La Mancha, Madrid, Universidad de Alcalá-Universidad de Castilla-La Mancha.

COMISIONES OBRERAS DE CASTILLA-LA MANCHA, (2004): La inmigración en Castilla-La Mancha. Una radiografía en 2003, Albacete, Altabán.

COMISIONES OBRERAS DE CASTILLA-LA MANCHA, (2007): Tras la primera ola, Albacete, Altabán.

CONSEJERÍA DE EDUCACIÓN Y CIENCIA, (2006): Modelo de Educación Intercultural y Cohesión Social de Castilla-La Mancha, Toledo, Consejería de Educación y Ciencia.

CONSEJERÍA DE TRABAJO Y EMPLEO, (2006): Plan Regional para la Integración Laboral de Inmigrantes en Castilla-La Mancha 2006-2007, Toledo, Consejería de Trabajo y Empleo.

CONSEJO ECONÓMICO Y SOCIAL DE EXTREMADURA, (2003): La inmigración en Extremadura, Badajoz, Consejo Económico y Social.

CONSEJO ESCOLAR DE CASTILLA-LA MANCHA, (2004): Inmigración y educación. La escolarización del alumnado inmigrante en Castilla-La Mancha, Toledo, Consejo Escolar de Castilla-La Mancha.

DECRETO 3/2004, de 20 de enero, de Régimen Jurídico de las viviendas con protección pública, en Diario Oficial de Castilla-La Mancha, no 10, 23 de enero de 2004.

DECRETO 3/2008, de 8 de enero, de la Convivencia Escolar en Castilla-La Mancha, en Diario Oficial de Castilla-La Mancha, ${ }^{\circ}$ 9, de 11 de enero de 2008.

DECRETO 338/2008, de 4 de noviembre, por el que se crea y regula el Foro Regional para la integración de la población inmigrante en Castilla-La Mancha, en Diario Oficial de Castilla-La Mancha, $\mathrm{n}^{\mathrm{o}} 230$, de 7 de noviembre de 2008.

DECRETO 92/2004, de 11 de mayo, por el que se establece la Estructura Orgánica y las Competencias de la Consejería de Trabajo y Empleo, en Diario Oficial de Castilla-La Mancha, $\mathrm{n}^{\circ}$ 81, de 14 de mayo de 2004.

DIRECCIÓN DE TRABAJO E INMIGRACIÓN, (2008): Memoria Técnica sobre la Gestión del Fondo 2007 en Castilla-La Mancha para la Acogida, la Integración y el Refuerzo Educativo de Inmigrantes, Toledo, Consejería de Trabajo y Empleo.

DIRECCIÓN GENERAL DE SALUD PÚBLICA Y PARTICIPACIÓN, (2006): Guía de actuación en atención primaria para población inmigrante, Toledo, Consejería de Sanidad. 
JUNTA DE COMUNIDADES DE CASTILLA-LA MANCHA, (2002): $2^{\circ}$ Plan regional de Integración Social Castilla-La Mancha 2002-2005, Toledo, Consejería de Bienestar Social.

LA VERDAD, 31 de julio de 2007, "Un estudio confirma que la inmigración no supone un problema de salud pública".

MARTÍNEZ DE LIZARRONDO, A., (2008): "Políticas autonómicas de integración de inmigrantes: el ámbito de los Servicios Sociales" en Servicios Sociales y Política Social, 84: 9-25.

- (2009): "Los procesos de gestión, coordinación y evaluación de las políticas autonómicas de integración de inmigrantes" en Gestión y Administración de Políticas Públicas, 36.

MARTÍNEZ DE LIZARRONDO, A. y LAPARRA, M., (2008): "Las políticas de integración social de inmigrantes en España" en A. IZQUIERDO (comp.), Inmigración y exclusión social, FOESSA, Madrid.

RED DE LUCHA CONTRA LA POBREZA Y LA EXCLUSIÓN SOCIAL EN CASTILLA-LA MANCHA, (2006): La inmigración en Castilla-La Mancha. Informe 2006, Toledo, Red de Lucha contra la Pobreza y la Exclusión Social de Castilla-La Mancha.

RESOLUCIÓN 16.679, de 21 de julio de 1997, de la Subsecretaría, por la que se dispone la publicación de la Resolución de 4 de julio de la Presidenta del Instituto Nacional de Estadística y del Director General de Cooperación Territorial, por la que se dictan instrucciones técnicas a los Ayuntamientos sobre actualización del Padrón municipal, en BOE, 177, 25 de julio de 1997.

SERVICIO DE INTEGRACIÓN SOCIAL, (2008): Red Regional de Atención a Inmigrantes Año 2008, Consejería de Salud y Bienestar Social, Toledo.

VV.AA. (2006): Acuerdo por la Convivencia en los Centros Escolares de Castilla-La Mancha, Toledo, VV.AA.

\section{Breve currículo:}

\section{Antidio Martínez de Lizarrondo Artola}

Doctor en Trabajo Social y Profesor Asociado en la Universidad Pública de Navarra. Su principal línea de investigación se centra en las políticas de integración social para personas inmigrantes (tanto la colaboración en su diseño e implementación, como su evaluación). Entre sus principales publicaciones destacan los artículos: "Integración y políticas de integración" incluido en la obra Extranjeros en el purgatorio. Integración social de los inmigrantes en el espacio local, M. Laparra, (ed.), Barcelona, Bellaterra, pp. 21-60. El escrito en colaboración con M. Lapparra "Las políticas de integración social de inmigrantes en España" en Fundación FOESSA, VI Informe FOESSA sobre exclusión y desarrollo social en España, (2008a), Madrid, Fundación FOESSA. Y por último "Una mirada a los planes de integración de las Comunidades Autónomas" en L. Cachón y M. Laparra (comp.) (2008b): Inmigración y política social, Barcelona, Bellaterra. 